\title{
Measurement of the analyzing powers in pd elastic and $p n$ quasi-elastic scattering at small angles at ANKE-COSY
}

\section{S. Dymov* for the ANKE collaboration}

University of Ferrara and INFN, I-44100 Ferrara, Italy,

Laboratory of Nuclear Problems, JINR, RU-141980 Dubna, Russia

E-mail: s.dymovefz-juelich.de

\begin{abstract}
The analyzing powers in proton-deuteron elastic and proton-neutron quasi-elastic scattering have been measured at small angles using a polarized proton beam at the COSY storage ring incident on an unpolarized deuterium target. The data were taken at $796 \mathrm{MeV}$ and five higher energies from $1600 \mathrm{MeV}$ to $2400 \mathrm{MeV}$. The analyzing power in $p d$ elastic scattering was studied by detecting the low energy recoil deuteron in telescopes placed symmetrically in the COSY plane to the left and right of the beam whereas for pn quasi-elastic scattering a low energy proton was registered in one of the telescopes in coincidence with a fast scattered proton measured in the ANKE magnetic spectrometer. Though the experiment explores new domains, the results are consistent with the limited published information.
\end{abstract}

23rd International Spin Physics Symposium - SPIN2018 -

10-14 September, 2018

Ferrara, Italy

\footnotetext{
* Speaker.
} 


\section{Introduction}

The nucleon-nucleon $(N N)$ interaction is of great importance in any study of hadronic processes at intermediate energies. At such energies a full set of amplitudes may be extracted using a phase-shift analysis but this is obviously dependent on the availability of a reliable experimental data base. Proton-proton elastic scattering has been extensively studied in many laboratories worldwide and the wealth of spin-dependent quantities measured has allowed the extraction of $N N$ phase shifts in the isospin $\mathrm{I}=1$ channel up to almost $3000 \mathrm{MeV}[1,2]$. The situation is far less promising for the isoscalar channel where the much poorer neutron-proton data base only permits the $\mathrm{I}=0$ phase shifts to be evaluated up to at most $1300 \mathrm{MeV}$.

Small angle neutron-proton elastic scattering has been studied at COSY by measuring the interaction of a deuteron beam with a hydrogen target [3, 4]. However, in this case the maximum beam energy at COSY is about $1150 \mathrm{MeV} /$ nucleon. To go higher in energy, where $n p$ data are very scarce, measurements have to be performed using a proton beam incident on a deuterium target.

The differential cross section [5] and analyzing power [6] in proton-proton elastic scattering have been studied at COSY using the ANKE magnetic spectrometer. We present here the recently published results of ANKE measurements of the proton analyzing powers in elastic protondeuteron and quasi-elastic proton-neutron scattering [7].

\section{Experimental setup}

The experiment was carried out using the ANKE magnetic spectrometer [8] positioned inside the COSY storage ring [9] of the Forschungszentrum Jülich. Although the facility sketched in Fig. 1 was equipped with other elements, the only detectors used in this experiment were the forward detector (FD) and the silicon tracking telescopes (STT) [10].

Fast protons from small-angle proton-deuteron elastic scattering or quasi-elastic scattering on the constituent nucleons were measured in the FD in the range $4^{\circ}-10^{\circ}$ in laboratory polar angle $\left(\theta_{\mathrm{lab}}\right)$ and $160^{\circ}-200^{\circ}$ in azimuth $(\phi)$. The FD comprises a set of multiwire proportional and drift chambers and a two-plane scintillation hodoscope. In addition to their use for triggering, scintillators were also needed to measure the energy losses required for particle identification [11].

The two STT were installed in the COSY plane symmetrically inside the vacuum chamber to the left and right of the beam at distances of $3 \mathrm{~cm}$ from the deuterium cluster-jet target, which had a jet diameter of about $1 \mathrm{~cm}$ [12]. Each telescope consists of three position-sensitive silicon layers of $70 \mu \mathrm{m}, 300 \mu \mathrm{m}$, and $5 \mathrm{~mm}$ thickness and, in this configuration, covered laboratory polar angles $75^{\circ}<\theta_{\text {lab }}<140^{\circ}$. The acceptances of the STT in azimuth of $\pm 30^{\circ}$ were centred at $\phi=0^{\circ}$ and $\phi=180^{\circ}$ on the left and the right sides, respectively.

The ANKE experiment used a vertically polarized beam incident on an unpolarized target and the preparation of the beam and the measurement of its polarization were carried out in common with the studies of the analyzing powers in proton-proton elastic scattering [6]. The sign of the polarization was flipped at every beam injection at the beginning of the acceleration cycle. The beam polarization after acceleration was measured using a simplified version of the EDDA detector as a polarimeter [13]. The experiment was carried out at six proton kinetic energies, $T_{p}=796,1600$, 


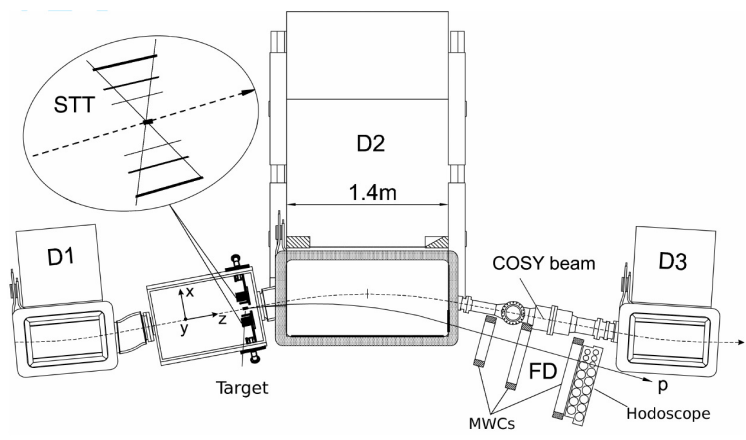

Figure 1: The ANKE spectrometer setup (top view), showing the positions of the deuterium clusterjet target, the silicon tracking telescopes (STT), and the forward detector (FD).

$1800,1965,2157$, and $2368 \mathrm{MeV}$. Cycles of $180 \mathrm{~s}$ or $300 \mathrm{~s}$ duration were used, with the last $20 \mathrm{~s}$ of each cycle being reserved for the beam polarization measurements.

\section{Analyzing power in proton-deuteron elastic scattering}

Proton-deuteron elastic scattering was cleanly identified and measured by detecting the deuteron in one of the STT without the use of the forward detector. The deuterons were identified via the energy loss in the STT, and the reaction was then selected through the missing mass criterion. With two STT placed symmetrically (left and right) around the target to form a two-arm polarimeter, the proton analyzing power in $p d$ elastic scattering could be measured in a completely analogous way to the measurements in $p p$ elastic scattering [6]. The asymmetry of $\vec{p} d$ elastic scattering was evaluated for each pair of successive cycles with beam polarizations up and down, using the cross-ratio method [14], which eliminates first-order systematic errors.

The angular dependence of the proton analyzing power in $\vec{p} d$ elastic scattering was determined from the STT-trigger data for all six beam energies and the results are shown in Fig. 2 in terms of the c.m. momentum transfer $q$. On general grounds the proton analyzing power ${ }^{1}$ is of the form of $q$ times a function of $q^{2}$ and the ANKE $796 \mathrm{MeV}$ results of Fig. 2 are well described by

$$
A_{y}^{p}(p d)=0.4714 q-0.0987 q^{3}+0.0077 q^{5}
$$

This form also reproduces very well the LAMPF data [15] provided that it is multiplied by a factor of 1.021 . This $2 \%$ difference is to be compared with the $3 \%$ precision in the beam polarization measurements with EDDA [13], which is our dominant systematic uncertainty, and the $2 \%$ systematic uncertainty in the LAMPF beam polarization [16].

The only obvious theoretical description of proton-deuteron elastic scattering data is the refined Glauber model of Platonova and Kukulin [17]. As in the original Glauber spinless work [18], there are contributions from single and double scattering that involve, respectively, the interaction

\footnotetext{
${ }^{1}$ We use a notation where $A_{y}^{p}(p d)$ is the proton analyzing power in $p d$ elastic scattering and $A_{y}^{p}(p n)$ is the same in $p n$ elastic scattering. The deuteron vector analyzing power in $d p$ elastic scattering is denoted by $A_{y}^{d}(d p)$.
} 


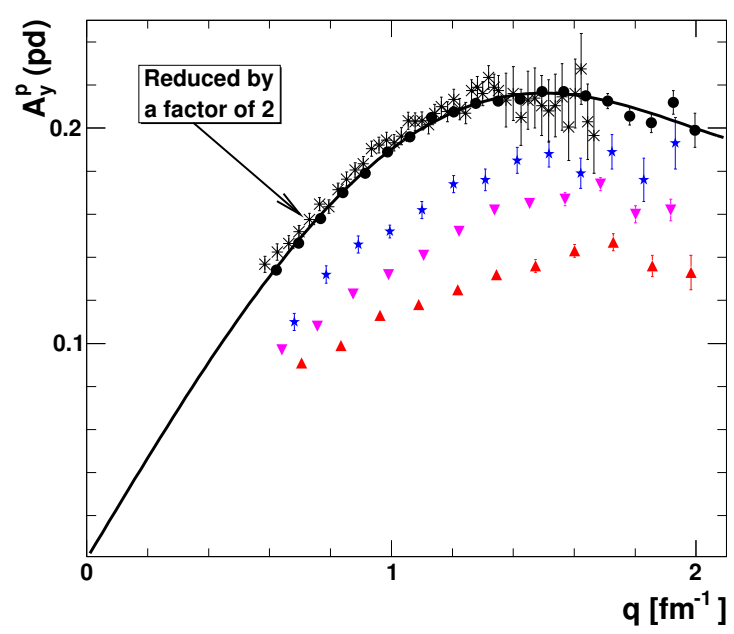

Figure 2: The proton analyzing power $A_{y}^{p}(p d)$ in proton-deuteron elastic scattering as a function of the c.m. momentum transfer $q$. ANKE data at $796 \mathrm{MeV}$ are shown by closed (black) circles, at $1600 \mathrm{MeV}$ by (blue) stars, at $1965 \mathrm{MeV}$ by (magenta) inverted triangles, and at $2368 \mathrm{MeV}$ by (red) triangles. Only statistical errors are shown and in general these are smaller than the symbol size. The fit of Eq. (3.1) to the ANKE data at $796 \mathrm{MeV}$ is shown by the continuous curve. The LAMPF data at this energy are shown by (black) crosses [15]. In order to increase the visibility of the higher energy points, both results and the curve at $796 \mathrm{MeV}$ are reduced by a factor of two.

with one or two nucleons in the deuteron. However, the nucleon-nucleon amplitudes used as input include all the five spin-dependent $p p$ and $p n$ terms so that predictions can be made of the polarization observables in $p d$ elastic scattering. Using the SAID $N N$ partial wave analysis [2], Platonova and Kukulin predicted the angular dependence of $A_{y}^{p}(p d)$ at $796 \mathrm{MeV}$ in the refined Glauber model [19]. Though the structure that they found is similar to that of the experimental data, their calculations underestimate the experimental data at low $q$ shown in Fig. 2. No reliable predictions could be made at our higher energies due to the lack of a $p n$ partial wave analysis above $1.3 \mathrm{GeV}$.

\section{Analyzing power in quasi-elastic proton-neutron scattering}

Events corresponding to the breakup reaction $p d \rightarrow p p n$ can be identified by measuring a fast proton in the forward detector and a slow one in one of the STT. These then provide a missing-mass distribution with the neutron peak well separated from the inelastic continuum and the background under the peak is only a few percent.

Due to the asymmetric acceptance of the FD, the count rate from the $p d \rightarrow p p n_{\text {spec }}$ reaction was found to be strongly suppressed kinematically compared with $p d \rightarrow p n p_{\text {spec }}$ when the slow proton was detected in the right-side STT. The count rate from quasi-free $p p$ is then expected to be less than $5 \%$ of that from quasi-free $p n$ even at the $796 \mathrm{MeV}$. At higher beam energies the limit reduces to below $3 \%$.

The quasi-free scenario is generally assumed to be realized when the momentum transfer from a beam particle to a scattered one $\left(p_{T}\right)$ is large compared with the "spectator" particle momentum 
$\left(p_{\text {spec }}\right)$, which should correspond to the Fermi momentum in the deuteron. This was achieved by selecting the data with the restriction $p_{T}>190 \mathrm{MeV} / c$ as well as $p_{\text {spec }} / p_{T}<0.5$.

The applicability of the "spectator" model was tested in the $796 \mathrm{MeV}$ data. Using the conservative limits mentioned above, values of the analyzing power were obtained that were in good agreement with the SAID SP07 [2] solution as well as with the data measured in Ref. [20] down to $\Theta_{c m}=17^{\circ}$, as shown in the Fig. 3.

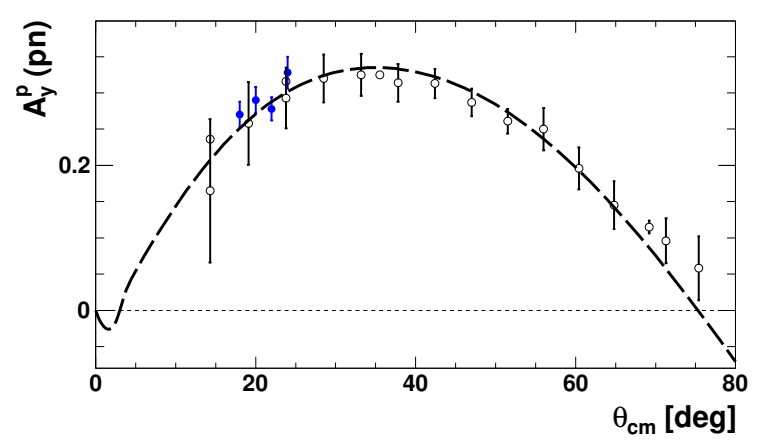

Figure 3: Analyzing power $A_{y}^{p}(p n)$ in quasi-elastic $\vec{p} n$ scattering at $T_{p} \approx 796 \mathrm{MeV}$. The open points are results from Ref. [20] as a function of the centre-of-mass scattering angle $\Theta_{c m}$. The blue solid points show results from the current experiment extracted under conditions discussed in the text. The predictions of the SAID SP07 partial wave solution [2] are shown by the dashed curve.

The values of the analyzing power generally decrease with increasing beam energy and the results presented in Fig. 4 illustrate the scale of the dependence. Despite the different experimental approach, the ANKE results at $2200 \mathrm{MeV}$ are fully consistent with data from Refs. [21, 22]. As was stressed already, the data base on $p n$ elastic scattering observables is insufficient to yield reliable partial wave solutions above about $1300 \mathrm{MeV}$. It is therefore not surprising that the SAID SP07 solution [2] does not describe well our new experimental data shown in Fig. 4. However, the SAID solution was recently updated to take into account the experimental data measured at COSYWASA [4]. Although it was asserted that the new AD14 solution [23] is still valid only up to $1300 \mathrm{MeV}$, it, nevertheless, gives predictions that are much closer to our $1600 \mathrm{MeV}$ data shown in Fig. 4 than those of SP07 [2].

\section{Conclusions}

We have measured the analyzing power in $\vec{p} d$ elastic and $\vec{p} n$ quasi-elastic scattering at $796 \mathrm{MeV}$ and at five energies from $1600 \mathrm{MeV}$ to $2400 \mathrm{MeV}$ at the COSY-ANKE facility. The results at $796 \mathrm{MeV}$ are consistent with published data to within the quoted uncertainties. The $\vec{p} d$ elastic measurements at $1600 \mathrm{MeV}$ and above were carried out for the first time at small angles and there is little $\vec{p} n$ elastic information at these higher energies.

The analyzing power in proton-neutron elastic scattering was studied in quasi-free conditions using a deuterium target. The validity of the empirical "spectator" approach was tested by comparing our result at $796 \mathrm{MeV}$ with data from other experiments. Good agreement was found between 


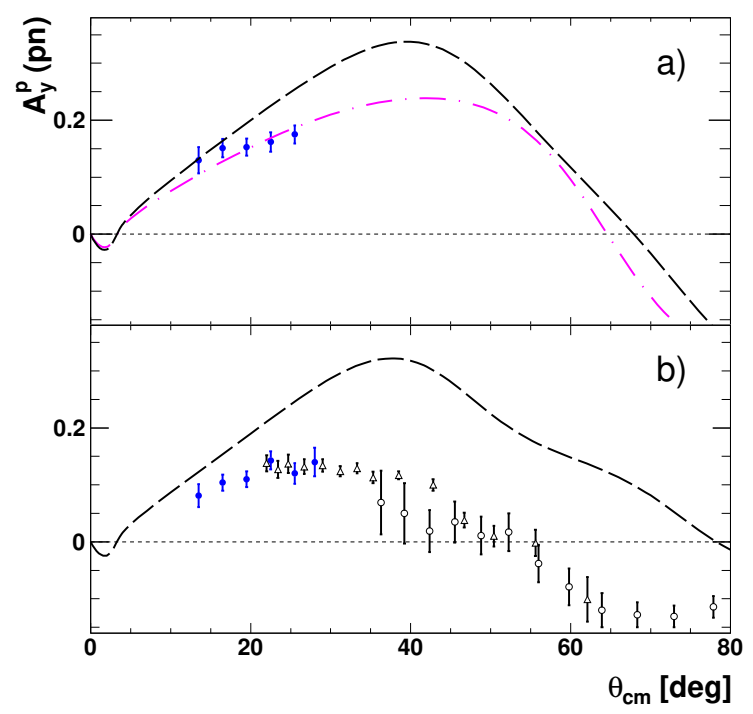

Figure 4: $A_{y}^{p}(p n)$ of quasi-free $\vec{p} n$ elastic scattering at (a) $1600 \mathrm{MeV}$ and (b) $2200 \mathrm{MeV}$ as function of the centre-of-mass scattering angle $\Theta_{c m}$. Solid (blue) points show the values obtained in the ANKE experiment whereas open (black) points are results taken from Ref. [21] (triangles) and Ref. [22] (circles). The magenta dot-dashed curve represents the new AD14 SAID solution at $1600 \mathrm{MeV}$ [23], though it should be noted that this energy is outside the stated range of validity of this solution. The dashed (black) curves in both panels illustrate the previous SAID solution [2], though it must be stressed that this also has limited validity above $1300 \mathrm{MeV}$.

our data at $2157 \mathrm{MeV}$ and the results from other experiments [21, 22]. Systematic uncertainties of our results were estimated to be about $5.5 \%$ at this energy and about $4 \%$ at others.

We are grateful to other members of the ANKE Collaboration for their help with this experiment and to the COSY crew for providing such good working conditions. This material is based upon work supported by the Forschungszentrum Jülich (COSY-FEE) and the Shota Rustaveli National Science Foundation Grant 09-1024-4-200.

\section{References}

[1] R.A. Arndt, I.I. Strakovsky, and R.L. Workman, Nucleon-nucleon elastic scattering to $3 \mathrm{GeV}$, Phys. Rev. C 62 (2000) 034005.

[2] R.A. Arndt, W.J. Briscoe, I.I. Strakovsky, and R.L. Workman, Updated analysis of NN elastic scattering to $3 \mathrm{GeV}$, Phys. Rev. C 76 (2007) 025209; http: / / gwdac . phys . gwu . edu.

[3] D. Mchedishvili, Studies of the neutron-proton charge-exchange amplitudes at COSY using the ANKE spectrometer, $\mathrm{PhD}$ thesis, University of Tbilisi 2013, available from collaborations.fz-juelich.de/ikp/anke/internal.shtml.

[4] P. Adlarson et al., Evidence for a New Resonance from Polarized Neutron-Proton Scattering, Phys. Rev. Lett. 112 (2014) 202301.

[5] D. Mchedlishvili et al., Measurement of the absolute differential cross section of proton-proton elastic scattering at small angles, Phys. Lett. B 755 (2016) 92. 
[6] Z. Bagdasarian et al., Measurement of the analyzing power in proton-proton elastic scattering at small angles, Phys. Lett. B 739 (2014) 152.

[7] S. Barsov et al., Measurement of the analyzing powers in $\vec{p} d$ elastic and $\vec{p} n$ quasi-elastic scattering at small angles, Eur. Phys. J. A $\mathbf{5 4}$ (2018) 225.

[8] S. Barsov et al., ANKE, a new facility for medium energy hadron physics at COSY-Jülich, Nucl. Instrum. Methods Phys. Res. A 462 (2001) 364.

[9] R. Maier et al., Cooler synchrotron COSY - Performance and perspectives, Nucl. Instrum. Methods Phys. Res. A 390 (1997) 1.

[10] R. Schleichert et al., A self-triggering silicon-tracking telescope for spectator proton detection, IEEE Trans. Nucl. Sci. 50 (2003) 301.

[11] S. Dymov et al., The forward detector of the ANKE spectrometer. Tracking system and its use in data analysis, Part. Nucl. Lett. 2(119) (2004) 40.

[12] A. Khoukaz et al., Systematic studies on hydrogen cluster beam production, Eur. Phys. J. D 5 (1999) 275.

[13] E. Weise, Kalibration des EDDA-Detektors als Polarimete: Messung der effektiven Analysierstärke, PhD thesis, University of Bonn, 2000.

[14] G.G. Ohlsen and P.W. Keaton Jr., Techniques for measurement of spin-1/2 and spin-1 polarization analyzing tensors, Nucl. Instrum. Methods 109 (1973) 41.

[15] F. Irom et al., Measurements of small angle elastic $\vec{p} d$ scattering at $796 \mathrm{MeV}$ using a recoil method, Phys. Rev. C 28 (1983) 2380.

[16] M.W. McNaughton et al., Spin correlation parameter $A_{n n}$ in pp elastic scattering at 796 MeV, Phys. Rev. C 23 (1981) 838.

[17] M.N. Platonova and V.I. Kukulin, Refined Glauber model versus Faddeev calculations and experimental data for pd spin observables, Phys. Rev. C 81 (2010) 014004.

[18] R.J. Glauber, Cross Sections in Deuterium at High Energies Phys. Rev. 100 (1955) 242.

[19] M.N. Platonova and V.I. Kukulin, in preparation.

[20] M.L. Barlett et al., Forward-angle elastic and quasielastic proton-nucleon cross sections and analyzing powers at 0.8 GeV, Phys. Rev. C 27 (1983) 682.

[21] R. Diebold et al., Measurement of the Proton-Neutron Elastic-Scattering Polarization from 2 to 6 GeV/c, Phys. Rev. Lett. 35 (1975) 632.

[22] Y. Makdisi et al., Analyzing Power in Large-Angle Proton-Neutron Elastic Scattering, Phys. Rev. Lett. 45 (1980) 1529.

[23] R.L. Workmann,W.J. Briscoe, and I.I. Strakovsky, Sensitivity of the COSY dibaryon candidate to $n p$ elastic scattering measurements, Phys. Rev. C 93 (2016) 045201. 